\title{
Coexistence of Granulomas and Metastatic Breast Carcinoma Deposit in an Axillary Lymph Node: A Rare Cytological Finding
}

\author{
Akanksha Bhatia, Prajwala Gupta* and Minakshi Bhardwaj
}

Department of Pathology, PGIMER, Dr. Ram Manohar Lohia Hospital, New Delhi, India

\section{Dear Sir}

Granulomatous reaction in malignancy, both intratumoral as well as in the draining lymph nodes have been reported in the literature. ${ }^{[1,2]}$ The main causes of granulomatous response in such cases include foreign body reaction to the necrotic tumor, therapy related granulomas or a coexistent systemic illness like tuberculosis and sarcoidosis. 3 However, in many cases a definitive cause is not found and the etiology remains obscure. We present here a case of invasive ductal carcinoma of the breast with metastatic deposit and granulomatous reaction in the ipsilateral axillary lymph node.

A 42 year old female presented to the hospital with lump in the left breast for the past 2 months. The patient had no systemic complaints. On examination, the swelling was firm, non tender and fixed; measured $1 \mathrm{x} 1 \mathrm{~cm}$ with irregular borders in the left upper outer quadrant of the left breast. Overlying skin was unremarkable. There was no history of nipple discharge. General examination revealed a lymph node swelling in the left axilla, which measured $1 \times 1 \mathrm{~cm}$ and was firm and mobile. Fine needle aspiration cytology (FNAC) was done from both the left breast and left axillary swellings. Cytology smears from the left breast swelling were highly cellular and showed malignant ductal cells arranged in dyscohesive, loose clusters and scattered singly
(Figure 1a). FNAC done from the left axillary swelling showed malignant ductal cells having similar morphology along with presence of epithelioid cell granulomas in a background of reactive lymphoid cells (Figure 1b). No necrosis was seen. Ziehl Neelsen staining for acid fast bacilli was negative. Overall cytological diagnosis given was invasive ductal carcinoma with coexistant granulomas and tumor deposit in the ipsilateral axillary lymph node.

Sir, a possibility of granulomatous reaction preceding the development of breast carcinoma may represent the likelihood of tuberculosis. The coexistence of breast cancer and tuberculosis within the breast as well as the axillary lymph node have been reported in the literature. ${ }^{[1,4]}$ Hence, in a country like India, where the incidence of tuberculosis is quite high, this coexistence may pose a diagnostic and clinical challenge. We ruled out tuberculosis in our case, by the absence of clinical features and smears being negative for acid fast tubercle bacilli. However, PCR based assays for tuberculosis maybe useful.

In cases of malignancy, the most frequent conditions associated with granulomas are tumor related granulomatous reaction. According to some authors, these granulomas result from a T-cell mediated immunological reaction to soluble antigens shed by the tumor. ${ }^{3}$ These granulomas are generally non caseating and may vary in size and number.

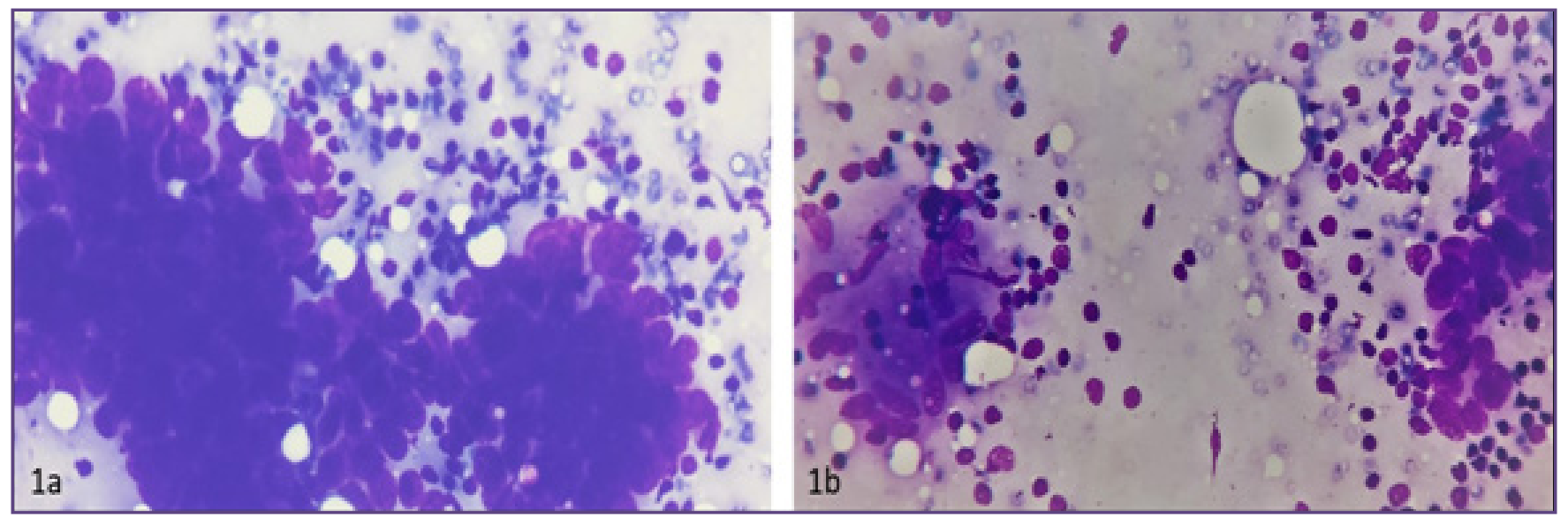

Fig. 1: a: FNAC from left breast swelling showing features of invasive ductal carcinoma (400X, Giemsa). b: FNAC from left axillary swelling showing tumor deposit with co-existent granuloma (400X, Giemsa). 
${ }^{5}$ Giant cells of both foreign body and Langhan's type and fibrinoid necrosis may also be present. In our case granuloma associated with tumor was not seen at the primary site of breast; rather at the metastatic lymph node.

Granulomas can rarely coexist with breast carcinoma at the metastatic site. Initial cytological evaluation with negative acid fast tubercular bacilli staining may be helpful in establishing an early and reliable diagnosis in such cases.

\section{Acknowlegement}

The authors declare no conflict of interest.

\section{References}

1. Khurram M, Tariq M, Shahid P. Breast cancer with associated granulomatous axillary lymphadenitis: a diagnostic and clinical dilemma in regions with high prevalence of tuberculosis. Pathol Res Pract 2007;203:699-704.

2. Alalshee T, Hamed T, Shafi SM. Granulomatous reaction associated with breast carcinoma: A report of two cases. Saudi J Med Med Sci 2014;2:120-2.

3. Bhatia A, Kumar Y, Kathpalia AS. Granulomatous inflammation in lymph nodes draining cancer: A coincidence or a significant association. Int J Med Med Sci 2009;1:13-16.

4. Akbulut S, Sogutcu N, Yagmur Y. Coexistence of breast cancer and tuberculosis in axillary lymph nodes: a case report and literature review. Breast Cancer Res Treat 2011;130:1037-42.

5. Munjal K, Jain VK, Agrawal A, Bandi PK. Co-existing tubercular axillary lymphadenitis with carcinoma breast can falsely over-stage the disease. Indian $\mathrm{J}$ Tuberc 2010;57:104-7.

*Corresponding author:

Dr. Prajwala Gupta Room no. 305, 3rd floor, OPD building, PGIMER, Dr. Ram Manohar Lohia Hospital, Baba Kharak Singh Marg,

New Delhi- 110001 INDIA

Phone: +91 9910306227

Email: prajwalagupta@gmail.com

Financial or other Competing Interests: None. 